\title{
Construction of recombinant adenovirus Ad-rat PLCg2-shRNA and successful suppression of PLCg2 expression in BRL-3A cells
}

\author{
X.G. Chen, Q.X. Lv and X.Q. Zhou \\ Animal Science and Technology School, \\ Henan University of Science and Technology, \\ Luoyang, Henan Province, China \\ Corresponding author: X.G. Chen \\ E-mail: cxguang1015@126.com \\ Genet. Mol. Res. 15 (2): gmr.15028029 \\ Received November 9, 2015 \\ Accepted January 22, 2016 \\ Published May 23, 2016 \\ DOI http://dx.doi.org/10.4238/gmr.15028029
}

\begin{abstract}
Phospholipase Cg2 (PLCg2) induces apoptosis of immune and tumor cells; however, it remains unclear whether PLCg2 promotes hepatocyte apoptosis during liver regeneration (LR). Therefore, to establish a framework for further exploring the function of PLCg2, we generated recombinant adenoviruses carrying a template encoding short hairpin (sh)-RNA targeting PLCg2 (Ad-PLCg2-shRNA), which were used to silence the expression of PLCg2 in BRL-3A cells. First, three pairs of PLCg2-shRNAs were designed, synthesized, and cloned into a shuttle vector, pHBAd-U6-GFP, after annealing. The recombinant shuttle plasmids were co-transfected with the backbone vector pHBAd-BHG into HK293 cells to package the recombinant Ad-PLCg2-shRNAs used to infect BRL-3A cells. Infection efficiency was monitored by observing the number of GFP-positive cells under a fluorescent microscope. To determine the recombinant adenoviruses
\end{abstract}


with the highest silencing efficiency, levels of $P L C g 2$ mRNA were evaluated by qRT-PCR. DNA sequencing confirmed that the correct shRNA coding sequences were inserted into the shuttle vectors and adenoviral plasmids. The titers of three recombinant adenoviruses were at least $1 \times 10^{10} \mathrm{PFU} / \mathrm{mL}$. The most effective adenoviral construct, with interference efficiency of $77 \%$, was determined by qRT-PCR. These results show that a recombinant adenovirus, Ad-PLCg2-shRNA, was developed and was effective at silencing the rat $P L C g 2$ gene. This construct may contribute to the study of PLCg2 in hepatocyte apoptosis during LR.

Key words: Phospholipase C gamma 2; Short hairpin RNA (shRNA); Recombinant adenovirus; BRL-3A cell; Rat

\section{INTRODUCTION}

Liver regeneration (LR) is a precisely controlled proliferative response of differentiated cells, which ultimately ends when liver mass and functions are sufficient for the organism (Rychtrmoc et al., 2012). Generally, LR occurs in three phases; initiation, proliferation, and termination (Corlu and Loyer, 2012). Of these, the termination phase is essential for successful LR, although this has not been as well studied as the initiation phase. Evidence suggests that there is a greater number of hepatocytes in regenerating liver than in normal liver (Miyaoka et al., 2012). At the end of LR, the liver mass needs to be accurately adjusted to match the prehepatectomy mass (Michalopoulos, 2010). Indeed, using partially hepatectomized rats, studies have detected a small wave of hepatocyte apoptosis at the end of LR in this species, which helps to correct the excess cell number and reveals the importance of apoptosis in the termination of LR (Michalopoulos, 2007). Previous observations have indicated that the end of LR requires the combined effects of both activin A and TGF-b1 (transforming growth factor-b1) to control hepatocyte mass by inhibiting hepatocyte proliferation and inducing hepatocyte apoptosis (Chen et al., 2014). However, many of the cytokines and growth factors necessary for proper termination have yet to be identified.

In our previous study on genome-wide gene expression changes in rat regenerating liver, phospholipase $\mathrm{C}$-gamma 2 ( $P L C g 2)$ was selected as an important LR-related gene, mainly due to its significant upregulation at the end of LR. Phospholipase Cg2 belongs to the phospholipase $\mathrm{C}$ enzyme family, which catalyzes the hydrolysis of phosphatidylinositol 4,5-bisphosphate (PIP2) to generate inositol 1,4,5-trisphosphate (IP3) and diacylglycerol (DAG), both of which act as second messengers to regulate multiple cellular responses (Pierce et al., 2002). IP3 released into the cytoplasm can mobilize calcium from the ER and subsequently trigger calcium signaling. The released calcium and DAG work together to activate protein kinase C (PKC) (Sherrill et al., 2009), which then phosphorylates a series of downstream molecules, subsequently regulating a large array of cellular processes, including inflammation, cell proliferation, differentiation, and development (Alberts et al., 2002; Xu et al., 2015). For instance, Hashimoto et al. (2000) reported that PLCg2-deficient mice exhibit impaired B-cell development, and many observations have also demonstrated the importance of PLCg2 in apoptosis. For example, in a study 
by Tomlinson et al. (2001), PLCg2 was found to be essential for the Bruton's tyrosine kinase (BTK):ER-induced apoptosis response. Zhang et al. (2003) also found that PLCg2 was significantly upregulated during TPA (12-O-tetradecanoylphorbol-1, 3-acetate)induced apoptosis in gastric cancer cells, suggesting that PLCg2 has an essential role in the induction of apoptosis. However, little is known about the contribution of PLCg2 to the induction of apoptosis during LR.

In order to develop tools necessary for $P L C g 2$ silencing, this study describes the AdMax adenovirus vector system for the construction of a recombinant $P L C g 2$ small interfering RNA (siRNA) adenovirus. Because siRNAs only affect target genes, these small molecules have become a widely used tool for gene silencing, and downregulate the expression of specific genes with perfect complementary sequence. In this way, siRNAs may have potential therapeutic activity against human diseases (Castanotto and Rossi, 2009; Naito and Ui-Tei, 2012). RNAi is a way of regulating genes and is a powerful tool for elucidating target gene function in mammalian cells (Ketting, 2011).

To study the effect of $P L C g 2$ gene expression on apoptosis during LR, we constructed recombinant shuttle adenoviruses carrying a short hairpin RNA (shRNA) targeting the rat $P L C g 2$ gene. These vectors were co-transfected with backbone vector pHBAd-BHG into HK293 cells to package and amplify recombinant adenoviruses. A high-titer adenovirus was selected to infect rat BRL-3A cells. To evaluate the efficiency of recombinant adenoviruses at silencing, the level of $P L C g 2$ mRNA was measured in infected BRL-3A cells using qRT-PCR. These findings provide strong support for further study on the role of PLCg2 in hepatocyte apoptosis and in the termination of LR.

\section{MATERIAL AND METHODS}

\section{Materials}

The BRL-3A cell line was kindly provided by Professor Cunshuan Xu (Key Laboratory for Cell Differentiation Regulation, Henan Normal University). HK293 cells, shuttle plasmid pHBAd-U6-GFP, and backbone vector pHBAd-BHG were purchased from Hanbio Co., Ltd. (Shanghai, China). Escherichia coli DH5a cells were purchased from Invitrogen (USA). DMEM (Dulbecco's Modified Eagle's Medium) (high glucose), LB culture medium, fetal bovine serum (FBS), and trypsin were purchased from GIBCO (USA). Restriction endonucleases (BamHI, EcoRI), T4 ligase, and DNA ladder were purchased from Fermentas (USA). Plasmid DNA and gel extraction kits were from CWBIO Company (Beijing, China) and Axygen (USA), respectively.

\section{Design and synthesis of shRNA sequences targeting rat PLCg2}

First, based on the principle of siRNA design and the full-length CDS of the rat PLCg2 gene (NM_017168.1), three siRNA oligonucleotide sequences targeting different sites of $P L C g 2$ were designed using a Promega siRNA target designer software (Promega Corp., USA). Next, based on the siRNA sequences, three pairs of single-stranded shRNA sequences were designed to incorporate into the shuttle plasmid pHBAd-U6-GFP, and were synthesized by Hanbio Co., Ltd. (Shanghai, China). 


\section{Construction and identification of recombinant shuttle plasmid pHBAd-U6-PLCg2 shRNA}

The adenovirus shuttle vector pHBAd-U6-GFP used in our study was linearized via double digestion with EcoRI and BamHI (Fermentas) and extracted from an agarose gel with a gel extraction kit (CWBIO, Beijing). Next, three pairs of single-stranded shRNAs were diluted to $100 \mu \mathrm{M}$ for renaturation. The renaturation conditions were $95^{\circ} \mathrm{C}$ for $10 \mathrm{~min}, 90^{\circ} \mathrm{C}$ for $10 \mathrm{~s}$, and then a gradient step where the temperature decreased by $20^{\circ} \mathrm{C}$ every $10 \mathrm{~min}$ until it reached $15^{\circ} \mathrm{C}$. The three synthesized double-stranded DNAs were named PLCg2-shRNA. PLCg2-shRNA was ligated overnight into the shuttle plasmid pHBAd-U6-GFP at a 3:1 ratio using T4 DNA ligase at $4^{\circ} \mathrm{C}$. Finally, ligation products were transformed into E. coli DH $5 \alpha$ competent cells using the thermal excitation method. The transformed cells were grown in LB medium containing $50 \mu \mathrm{g} / \mathrm{mL}$ ampicillin overnight at $37^{\circ} \mathrm{C}$. Positive colonies were selected and propagated for plasmid DNA preparation. To verify that cloning was successful, colony sequencing was performed by Shanghai Sunny Biotechnology Co., Ltd. The positive clones were named pHBAd-U6-PLCg2 shRNA1, pHBAd-U6-PLCg2 shRNA2, and pHBAd-U6PLCg2 shRNA3.

\section{Packaging and propagation of recombinant adenovirus Ad-PLCg2-shRNA}

Prior to infection, HK293 cells were seeded in Petri dishes at $37^{\circ} \mathrm{C}$ until they reached a logarithmic phase. Using LipoFiter ${ }^{\mathrm{TM}}$ transfection reagent purchased from Hanbio Co, Ltd. (Shanghai, China), the shuttle vector, pHBAd-U6-PLCg2-shRNA, and backbone vector, pHBAd-BHG, were co-transfected into HK293 cells. The transfected HK293 cells were incubated for $6 \mathrm{~h}$ and then the medium was replaced with fresh DMEM containing FBS. After 10 days of culture, a typical grape-like aggregate morphology that is caused by cytopathic effect (CPE) was observed in the majority of infected cells. The cells were collected, frozen, and thawed thrice at temperatures between $-70^{\circ}$ and $37^{\circ} \mathrm{C}$, centrifuged at $1500 \mathrm{~g}$ for $5 \mathrm{~min}$ at $25^{\circ} \mathrm{C}$. After sedimentation of cell debris, the supernatant contained the first-generation $\mathrm{Ad}$ vector expressing PLCg2 shRNA (Ad-PLCg2-shRNA).

Two-milliliters of first-generation Ad-PLCg2-shRNA was used to infect HK293 cells grown on 10-mm Petri dishes for amplification. After two rounds of propagation, high-titer recombinant adenovirus (Ad-PLCg2-shRNA) was harvested and stored at $-80^{\circ} \mathrm{C}$ until use.

\section{Determination of viral titer}

To calculate the 50\% tissue culture infective dose (TCID50) of Ad-PLCg2-shRNA, HK293 cells were seeded into clear, flat-bottomed 96-well plates at a density of $1 \times 10^{4}$ cells/ well in $100 \mu \mathrm{L}$ DMEM with 10\% FBS. Eight replicate wells were used for each dilution. Tenfold serial dilutions of adenoviral samples were prepared from rows $\mathrm{A}$ to $\mathrm{H}\left(10^{-6}\right.$ to $\left.10^{-13}\right)$, and $100 \mu \mathrm{L}$ diluted viral samples was inoculated in the corresponding wells. Columns 11 and 12 were used as no-virus controls. The plates were incubated at $37^{\circ} \mathrm{C}, 5 \% \mathrm{CO}_{2}$ for 10 days. On the 11th day, each well was examined for evidence of CPE. For each dilution, the number of wells positive for CPE was scored. Karber's method was employed to calculate the $\mathrm{TCID}_{50}$, which was scaled up to give the $\mathrm{TCID}_{50}$ per milliliter. 


\section{Evaluation of the PLCg2 gene silencing by a recombinant adenovirus expressing PLCg2-targeted shRNA}

Levels of rat $P L C g 2$ mRNA in the transfected BRL-3A cells were measured by qRTPCR. BRL-3A cells $\left(1 \times 10^{5}\right.$ cells $\left./ \mathrm{mL}\right)$ were seeded onto 6-well plates in DMEM supplemented with $10 \% \mathrm{FBS}$, then incubated overnight at $37^{\circ} \mathrm{C}$ in $5 \% \mathrm{CO}_{2}$. Before transfection, the medium was replaced with fresh medium of the same composition. Upon reaching $80-90 \%$ confluence, BRL-3A cells were infected with recombinant adenoviruses at a multiplicity of infection (MOI) of 50. The efficiency of infection was evaluated by fluorescence microscopy after $48 \mathrm{~h}$ of infection. Ad-GFP was used as the positive control.

The cells were then harvested and total RNA was extracted, purified, and reverse transcribed into cDNA. Based on the $P L C g 2$ gene sequence, primer sequences for qRT-PCR were designed using the Primer 5.0 software. GAPDH was used as an internal reference gene. qRT-PCR primers specific for PLCg2 and GAPDH are listed in Table 1. These primer sequences were synthesized by Shanghai Sunny Company (China). qRT-PCR was performed in a $20-\mu \mathrm{L}$ reaction mixture containing $10 \mu \mathrm{L} 2 \mathrm{X}$ Real-time PCR Master Mix, $0.8 \mu \mathrm{L} 10 \mathrm{M}$ each primer, $1 \mu \mathrm{L}$ template cDNA, and $8.2 \mu \mathrm{L} \mathrm{ddH}_{2} \mathrm{O}$ water. The amplification conditions were as follows: $95^{\circ} \mathrm{C}$ for $3 \mathrm{~min}$, and 40 cycles of $95^{\circ} \mathrm{C}$ for $15 \mathrm{~s}, 60^{\circ} \mathrm{C}$ for $15 \mathrm{~s}$, and $72^{\circ} \mathrm{C}$ for $20 \mathrm{~s}$.

Table 1. PLCg2 and GAPDH primer sequences for qRT-PCR.
\begin{tabular}{l|l}
\hline Gene & Primer sequence \\
\hline PLC 2 & F 5 '-CTGGCAACCGACTCAAAGGA-3' \\
& R: 5'-GCTGATGCTGTTTCTTCGGG-3' \\
\hline GAPDH & F: 5'-GCTGGTGCTGAGTATGTCGT-3' \\
& R: 5'-TCACAAACATGGGGGCATCA-3' $^{\prime}$ \\
\hline
\end{tabular}

\section{RESULTS}

\section{shRNA sequence targeting the rat $P L C g 2$ gene}

Based on three siRNA sequences, six corresponding single-stranded shRNA sequences targeting the PLCg2 gene were designed and synthesized, as shown in Table 2. PLCg2shRNA was designed based on the principle that shRNA will form a hairpin structure with an intervening TTCAAGAGA loop; several extra nucleotides were added to generate EcoRI and BamHI sites on the 5'-end of the forward and reverse chains, respectively, which would be convenient for insertion into the shuttle vector pHBAd-U6-GFP.

\begin{tabular}{|c|c|c|}
\hline \multicolumn{2}{|c|}{ RNAi targets (5'-3') } & shRNA sequences $\left(5^{\prime}-3^{\prime}\right)$ \\
\hline siRNA1 & GCTGCGGAAACAGATTTATTCAGTA & $\begin{array}{l}\text { F: AATTCGCTGCGGAAACAGATTTATTCAGTAttcaagagaTACTGAATAAATC } \\
\text { TGTTTCCGCAGCtttttg } \\
\text { R: GATCCAAAAAAGCTGCGGAAACAGATTTATTCAGTATctcttgaaTACTGAA } \\
\text { TAAATCTGTTTCCGCAGCg }\end{array}$ \\
\hline siRNA2 & GCCAGCTTCGTGAGAAGATCA & $\begin{array}{l}\text { F: AATTCGCCAGCTTCGTGAGAAGATCAttcaagagaTGATCTTCTCACGAA } \\
\text { GCTGGCtttttg } \\
\text { R: GATCCAAAAAAGCCAGCTTCGTGAGAAGATCATctcttgaaTGATCTTCTCA } \\
\text { CGAAGCTGGCg }\end{array}$ \\
\hline siRNA3 & GAGACAAAGGCAGACAGCATTGTCA & $\begin{array}{l}\text { F: AATTCGAGACAAAGGCAGACAGCATTGTCAttcaagagaTGACAATGCTG } \\
\text { TCTGCCTTTGTCTCtttttg } \\
\text { R: GATCCAAAAAAGAGACAAAGGCAGACAGCATTGTCATctcttgaaTGACA } \\
\text { ATGCTGTCTGCCTTTGTCTCg }\end{array}$ \\
\hline
\end{tabular}




\section{Construction of recombinant shuttle plasmid pHBAd-U6-PLCg2 shRNA}

Three annealed double-stranded shRNAs were individually cloned into the linearized shuttle vector pHBAd-U6-GFP, which had been double-digested with EcoRI and BamHI, thus generating the recombinant plasmids pHBAd-U6-PLCg2-shRNA1, -shRNA2, and -shRNA3. Sequencing was carried out to verify the successful construction of the recombinant shuttle plasmid. As shown in Figure 1, the results were consistent with the forward strand sequences of the three double-stranded shRNAs designed in this study, confirming the successful cloning of pHBAd-U6-PLCg2-shRNA.
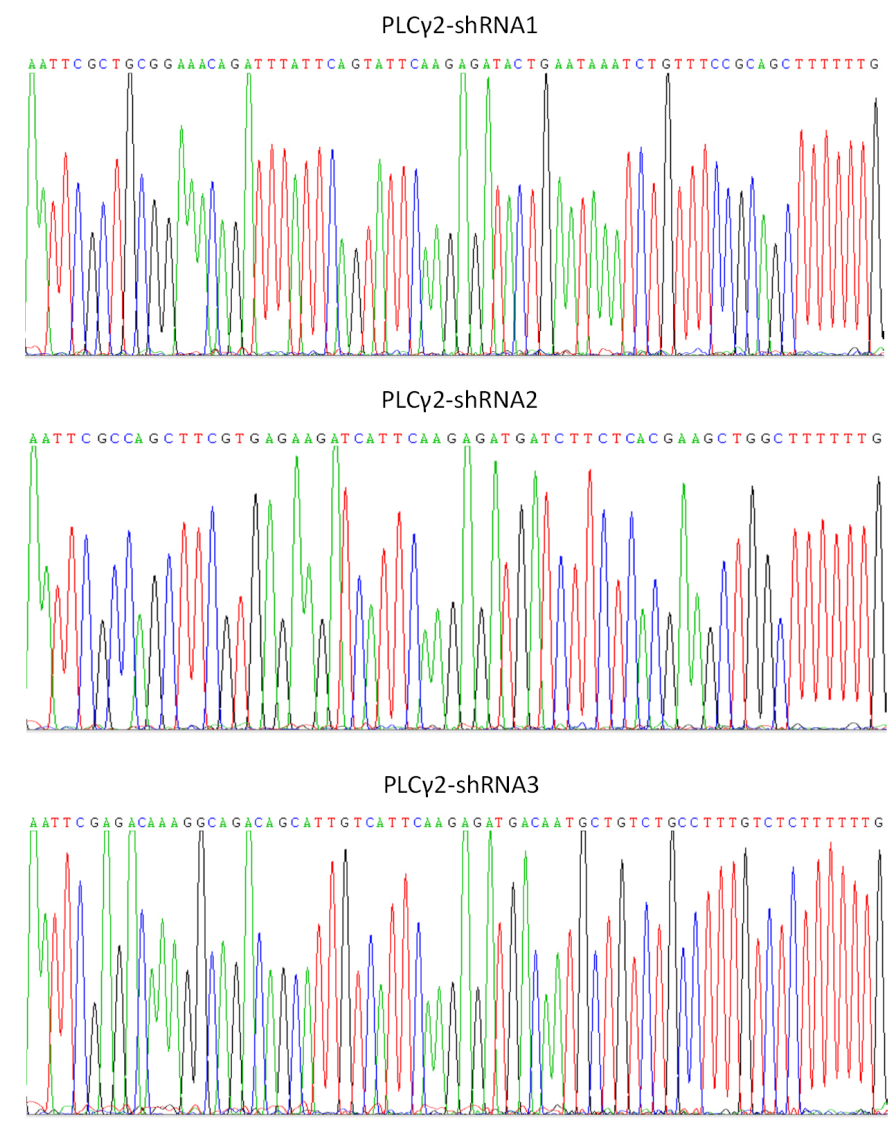

Figure 1. Sequence of the recombinant shuttle plasmid, pHBAd-U6-PLCg2-shRNA.

\section{Packaging of recombinant adenoviruses carrying rat PLCg2-shRNA}

pHBAd-PLCg2 shRNA1, -shRNA2, and -shRNA3 were separately co-transfected with the adenovirus backbone vector pHBAd-BHG into HK293 cells. After 10 days of transfection, some cells began to display the typical cytopathic phenotype, showing characteristic rounded giant cells, cell migration in the medium, and the appearance of grape-like lesions (Figure 2), demonstrating the successful packaging of recombinant adenovirus (Ad-PLC $\gamma$ shRNA). 
Adenoviruses were collected before cells ruptured and then used to infect HK293 cells twice, giving rise to high-titer adenovirus. Results of the TCID50 assay revealed that the titers of Ad-PLCg2-shRNA1, -shRNA2, and -shRNA3 were $2 \times 10^{10}, 1 \times 10^{10}$, and $1.58 \times$ $10^{10} \mathrm{PFU} / \mathrm{mL}$, respectively. An MOI of 50 for Ad-PLCg2-shRNA was found to be optimal for infecting rat BRL-3A cells.

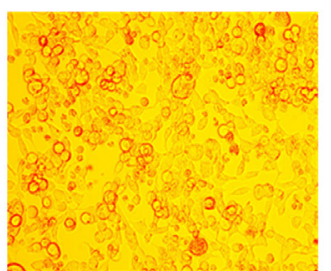

Ad-GFP

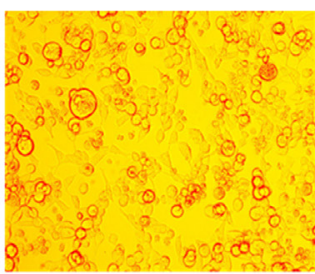

PLCV2-ShRNA1

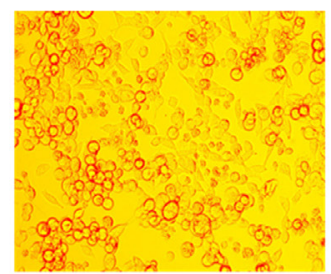

PLCV2-ShRNA2

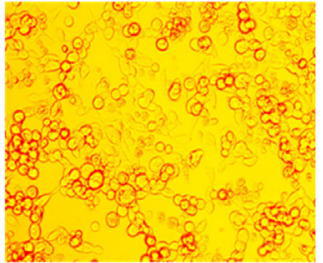

PLCV2-ShRNA3

Figure 2. Cytopathic effect of HK293 cells during the packaging of recombinant adenovirus.

\section{Infection efficiency of recombinant adenoviral vectors}

After $36 \mathrm{~h}$ of infection, the infection efficiency of Ad-PLCg2-shRNA in BRL-3A cells was monitored by fluorescence microscopy. As shown in Figure 3, there was a high percentage of fluorescent cells in both the Ad-GFP and Ad-PLCg2-shRNA groups, indicating that the three recombinant vectors efficiently infected BLR-3A cells without affecting normal cell viability. In addition, in contrast to the negative control group, Ad-PLCg2-shRNA3 showed the highest efficiency.

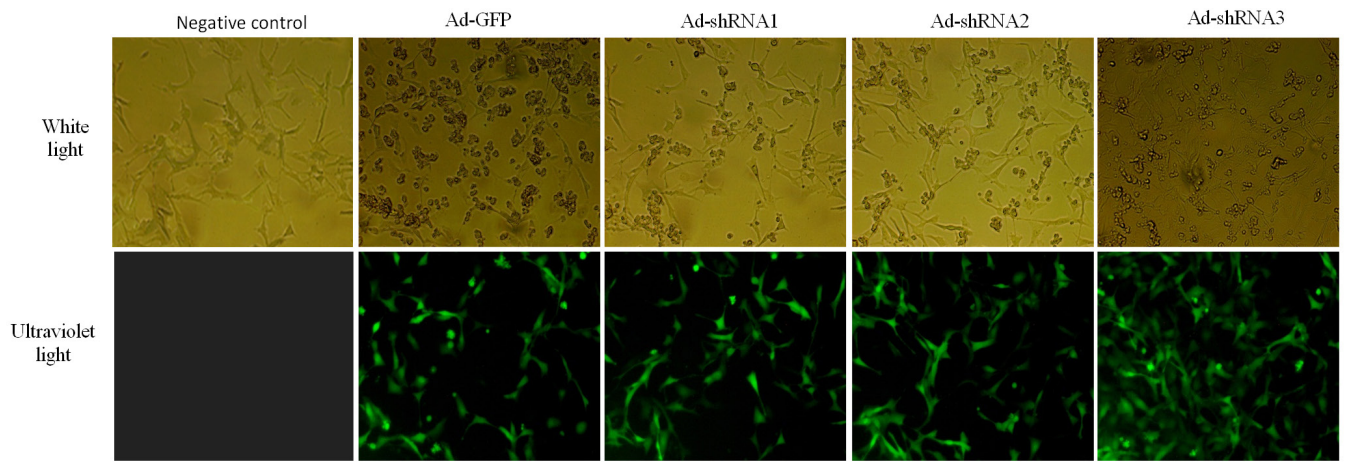

Figure 3. GFP (green fluorescent protein) expression in BRL-3A cells infected with Ad-GFP or recombinant adenovirus Ad-PLCg2-shRNA visualized under a fluorescence microscopy.

\section{Ad-PLCg2-shRNA silences $P L C g 2$ gene expression in BRL-3A cells}

To quantify the interference efficiency of recombinant adenovirus, total RNA from BRL-3A cells was extracted and purified. qRT-PCR was performed to analyze PLCg2mRNA expression. The results showed that compared with the Ad-GFP group, PLCg2 mRNA 
expression was inhibited by Ad-PLCg2-shRNA1, -shRNA2, -shRNA3 by 1, 69, and 77\%, respectively, suggesting that Ad-PLCg2-shRNA3 was the most effective at silencing the PLCg2 gene (Figure 4).

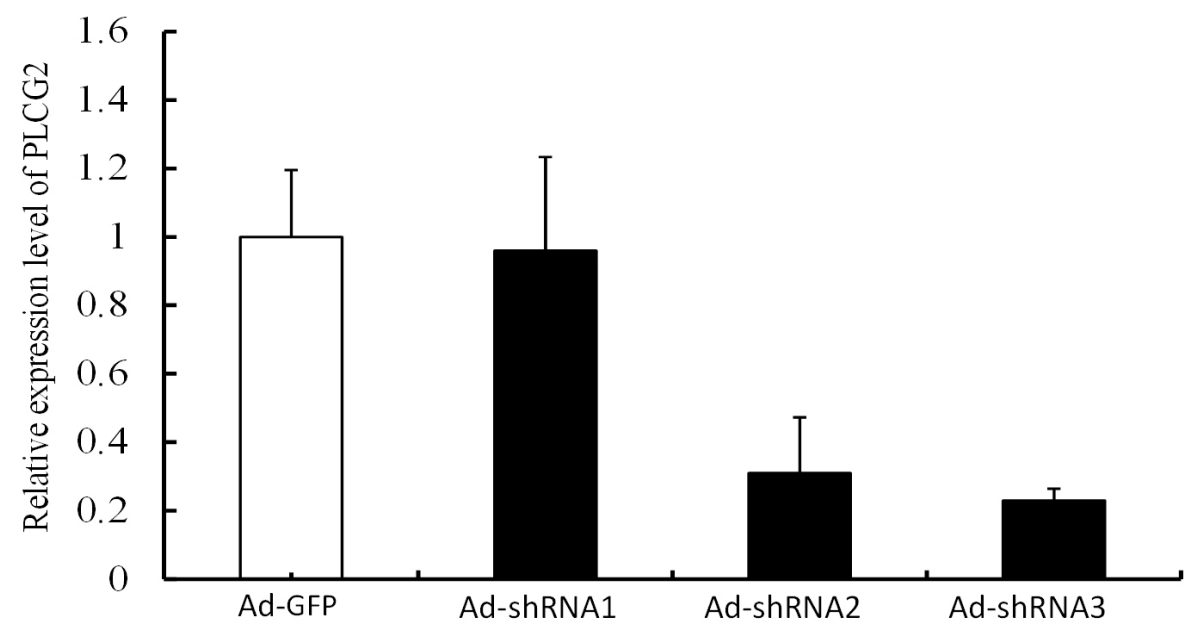

Figure 4. Relative level of PLCg2 mRNA in infected BRL-3A cell lines.

\section{DISCUSSION}

LR is an extremely complex process that is controlled by both positive and negative regulators (Böhm et al., 2010; Kang et al., 2012). To date, studies on LR have focused mainly on the positive control mechanisms, and the negative control mechanisms are not well studied or understood. This is especially true at the end of regeneration, when the liver mass is accurately adjusted to match the pre-surgery mass (Apte et al., 2009) via apoptosis of excess cells. When investigating gene expression profiling during rat LR, we selected PLCg2 as a gene of interest because of its significant upregulation during the termination of LR.

PLCg2 is a member of the PLC family, and is normally located in cytoplasm when the cell is in an inactive state. Once activated, this molecule translocates to the plasma membrane where it hydrolyzes PIP2 to form DG and IP3. These then activate downstream signaling pathways thus regulating various biological events, such as cell growth and differentiation (Ozdener et al., 2002). Recently, several studies have identified PLCg2 as an important regulator of apoptosis, and it has been reported to induce the apoptosis of gastric cancer cells (Zhang et al., 2003).

In order to study this gene during the termination of LR, a recombinant adenovirus vector that interferes with PLCg2 expression was generated in the present study. Several vectors have been used to deliver and express siRNA to knockdown the expression of target genes. This is dependent on high delivery efficiency, a rapid propagation period, high viral titers, large-fragment cloning capacity, and convenient storage. Adenovirus vectors have been extensively used in gene therapy, vaccine therapy, and in basic biology research (Ura et al., 2014). By combining RNAi technology with a viral gene delivering system, we built viral delivery vectors expressing PLCg2 shRNA. As a result, PLCg2 shRNA was efficiently 
transfected into BRL-3A cells via an adenoviral vector. Results of qRT-PCR showed that Ad-PLCg2-shRNA3 reduced the mRNA level of PLCg2 by 77\%, indicating the successful construction of a recombinant adenovirus expressing rat PLCg2 shRNA.

In conclusion, a recombinant Ad-PLCg2-shRNA vector was successfully constructed in the present study, which remarkably reduced PLCg2 expression in BRL-3A cells. As a tool for selectively silencing genes, Ad-PLCg2-shRNA represents a first step in the in-depth study of PLCg2 in hepatocyte apoptosis during LR.

\section{Conflicts of interest}

The authors declare no conflict of interest.

\section{ACKNOWLEDGMENTS}

Research supported by the National Natural Science Foundation of China (\#31401209). The authors kindly thank Professor Cunshuan Xu for his hearty support.

\section{REFERENCES}

Alberts B, Lewis J, Raff M, Roberts K, et al. (2002). Molecular Biology of the Cell (4th eds.). New York: Garland Science. Apte U, Gkretsi V, Bowen WC, Mars WM, et al. (2009). Enhanced liver regeneration following changes induced by hepatocyte-specific genetic ablation of integrin-linked kinase. Hepatology 50: 844-851. http://dx.doi.org/10.1002/ hep.23059

Böhm F, Köhler UA, Speicher T and Werner S (2010). Regulation of liver regeneration by growth factors and cytokines. EMBO Mol. Med. 2: 294-305. http://dx.doi.org/10.1002/emmm.201000085

Castanotto D and Rossi JJ (2009). The promises and pitfalls of RNA-interference-based therapeutics. Nature 457: 426433. http://dx.doi.org/10.1038/nature 07758

Chen L, Zhang W, Liang HF, Zhou QF, et al. (2014). Activin A induces growth arrest through a SMAD- dependent pathway in hepatic progenitor cells. Cell Commun. Signal. 12: 18-32. http://dx.doi.org/10.1186/1478-811X-12-18

Corlu A and Loyer P (2012). Regulation of the g1/s transition in hepatocytes: involvement of the cyclin-dependent kinase cdk1 in the DNA replication. Int. J. Hepatol. 2012: 1-17. http://dx.doi.org/10.1155/2012/689324

Hashimoto A, Takeda K, Inaba M, Sekimata M, et al. (2000). Cutting edge: essential role of phospholipase C-gamma 2 in B cell development and function. J. Immunol. 165: 1738-1742. http://dx.doi.org/10.4049/jimmunol.165.4.1738

Kang LI, Mars WM and Michalopoulos GK (2012). Signals and cells involved in regulating liver regeneration. Cells 1: 1261-1292. http://dx.doi.org/10.3390/cells1041261

Ketting RF (2011). The many faces of RNAi. Dev. Cell 20: 148-161. http://dx.doi.org/10.1016/j.devcel.2011.01.012

Michalopoulos GK (2007). Liver regeneration. J. Cell. Physiol. 213: 286-300. http://dx.doi.org/10.1002/jcp.21172

Michalopoulos GK (2010). Liver regeneration after partial hepatectomy: critical analysis of mechanistic dilemmas. Am. J. Pathol. 176: 2-13. http://dx.doi.org/10.2353/ajpath.2010.090675

Miyaoka Y, Ebato K, Kato H, Arakawa S, et al. (2012). Hypertrophy and unconventional cell division of hepatocytes underlie liver regeneration. Curr. Biol. 22: 1166-1175. http://dx.doi.org/10.1016/j.cub.2012.05.016

Naito Y and Ui-Tei K (2012). siRNA design software for a target gene-specific RNA interference. Front. Genet. 3: 1-12. http://dx.doi.org/10.3389/fgene.2012.00102

Ozdener F, Kunapuli SP and Daniel JL (2002). Expression of enzymatically-active phospholipase Cgamma2 in E. coli. $J$. Biochem. Mol. Biol. 35: 508-512. http://dx.doi.org/10.5483/BMBRep.2002.35.5.508

Pierce KL, Premont RT and Lefkowitz RJ (2002). Seven-transmembrane receptors. Nat. Rev. Mol. Cell Biol. 3: 639-650. http://dx.doi.org/10.1038/nrm908

Rychtrmoc D, Hubálková L, Víšková A, Libra A, et al. (2012). Transcriptome temporal and functional analysis of liver regeneration termination. Physiol. Res. 61 (Suppl 2): S77-S92.

Xu GG, Geng Z, Zhou XC, He YG, et al. (2015). Three branches of phospholipase C signaling pathway promote hepatocyte growth in rat liver regeneration. Genet. Mol. Res. 14: 5710-5723. http://dx.doi.org/10.4238/2015.May.29.3 
Sherrill JD, Stropes MP, Schneider OD, Koch DE, et al. (2009). Activation of intracellular signaling pathways by the murine cytomegalovirus G protein-coupled receptor M33 occurs via PLC-beta/PKC-dependent and -independent mechanisms. J. Virol. 83: 8141-8152. http://dx.doi.org/10.1128/JVI.02116-08

Tomlinson MG, Woods DB, McMahon M, Wahl MI, et al. (2001). A conditional form of Bruton's tyrosine kinase is sufficient to activate multiple downstream signaling pathways via PLC Gamma 2 in B cells. BMC Immunol. 2: 1-12. http://dx.doi.org/10.1186/1471-2172-2-4

Ura T, Okuda K and Shimada M (2014). Developments in Viral Vector-Based Vaccines. Vaccines (Basel) 2: 624-641. http://dx.doi.org/10.3390/vaccines2030624

Zhang B, Wu Q, Ye XF, Liu S, et al. (2003). Roles of PLC-gamma2 and PKCalpha in TPA-induced apoptosis of gastric cancer cells. World J. Gastroenterol. 9: 2413-2418. 